\title{
Increased efficiency of testicular tumor chemotherapy by ultrasound microbubble-mediated targeted transfection of siMDR1
}

\author{
YUN HE, YANG BI, XIAO-JUAN JI and GUANGHUI WEI \\ Department of Pediatric Surgery, Stem Cell Biology and Therapy Laboratory, Ministry of Education \\ Key Laboratory of Child Development and Disorders, Chongqing Stem Cell Therapy Engineering Technical Center, \\ The Children's Hospital of Chongqing Medical University, Chongqing 400014, P.R. China
}

Received March 23, 2015; Accepted April 21, 2015

DOI: $10.3892 /$ or.2015.4262

\begin{abstract}
The MDR1 gene encoding P-glycoprotein (P-gp) is an ATP-dependent drug efflux transporter and is related to drug resistance of yolk sac tumors. Drug resistence may be an important factor for the low efficiency of chemotherapy in the treatment of testicular tumors. P-gp, encoded by the MDR1 gene, is an ATP-binding cassette transporter. P-gp exhibits high expression in capillary endothelial cells of the testis and prevents the intracellular accumulation of chemotherapy agents in testicular tumor cells, resulting in drug resistance. In the present study, we aimed to use specific siRNA to silence the expression of the MDR1 gene and P-gp, leading to the reversal of multidrug resistance of testicular tumors and contributing a suitable condition for chemotherapy. Ultrasound microbubblemediated delivery is a safe and effective tool for gene delivery. In the present study, we demonstrated that ultrasound microbubble-mediated delivery effectively improved the siMDR 1 gene transfection in interstitial capillary endothelial cells of the testis, inhibited the expression of P-gp and increased daunorubicin accumulation. The testis tumor model was successfully constructed by injecting $1 \times 10^{7}$ yolk sac tumor cells in 3-weekold Sprague-Dawley rats. Ultrasound microbubble-mediated siMDR1 gene therapy improved the effect of chemotherapy on the testicular tumors. The testicular volume was reduced, the number of tumor cells within the testicular tissues decreased, and pathological changes were mostly recovered. Therefore, the present study indicated that ultrasound microbubble-mediated siMDR1 gene therapy in vivo reversed drug resistance by regulating $\mathrm{P}-\mathrm{gp}$ expression, providing a promising method for the treatment of testicular tumors.
\end{abstract}

Correspondence to: Dr Guanghui Wei, Department of Pediatric Surgery, The Children's Hospital of Chongqing Medical University, Chongqing 400014, P.R. China

E-mail: ghwei@cqmu.edu.cn

Key words: yolk sac tumor, ultrasound microbubble-mediated delivery, RNA interference, multiple drug resistance gene

\section{Introduction}

Testicular tumors are common malignant solid tumors of the urinary and reproductive system. Chemotherapy is the most common method to treat testicular cancer $(1,2)$. However, low therapeutic efficiency is a crucial factor affecting the therapeutic results (3). It has been found that P-glycoprotein (P-gp), as a multidrug resistance-associated protein, is highly expressed in capillary endothelial cells of the testis to form a biological barrier, blocking access of chemotherapy agents to tumor sites, playing an important role in the drug resistance of testicular tumors $(4,5)$. P-gp, encoded by the MDR1 gene, is a membrane protein that functions as an ATP-dependent efflux pump, pumping out water insoluble toxic substances from cells, and impeding the delivery of chemotherapeutic drugs to the testis $(6,7)$. Therefore, breaking through the blood-testis barrier and the reversal of drug resistance are key goals for the treatment of testicular tumors (8).

Thus, we aimed to use RNA interference technology to silence the expression of the MDR1 gene and inhibit expression of P-gp, to improve the responsiveness to chemotherapeutic drugs of testicular tumors in vitro $(9,10)$.

The first and most important problem of gene therapy is development of a safe and effective gene therapy carrier, as well as an effective way to delivery genes into target cells. However, commonly used traditional transfection methods are not satisfactorily translatable to in vivo conditions (11). Recent studies have shown that microbubble contrast agents can carry and release genes under ultrasonic action, promoting the in vitro and in vivo transfection of target genes. Ultrasound microbubble-mediated gene therapy is expected to be a safe, efficient and non-invasive gene therapy $(12,13)$.

In a previous study, we revealed that ultrasound microbubble-mediated gene delivery effectively assisted the entry of siMDR1 into L2RYC cells, inhibited the expression of the MDR1 gene and P-protein, as well as the efflux pump function of P-protein, increasing the aggregation of chemotherapy drugs in cells, thus resulting in a more effective antitumor effect (9). In the present study, we demonstrated that the siMDR gene-carrying polymer coated microbubble contrast agent reached the testis through intravenous injection, and 
destruction of microbubbles under the ultrasonic action successfully transfected the siMDR1 gene into rat testicular capillary endothelial cells. Silencing of MDR1 inhibited the expression and function of P-gp, so that the anticancer drug daunorubicin entered more easily into testicular tumors. Ultrasound microbubble-mediated gene therapy combined with RNAi, has a good application prospect in the treatment of testicular tumors.

\section{Materials and methods}

Cell culture and chemicals. The L2RYC cell line was obtained from ATCC (Manassas, VA, USA), and maintained in complete Dulbecco's modified Eagle's medium (DMEM) supplemented with $10 \%$ fetal bovine serum (FBS; HyClone, Logan, UT, USA), $100 \mathrm{U} / \mathrm{ml}$ penicillin and $100 \mu \mathrm{g} / \mathrm{ml}$ streptomycin at $37^{\circ} \mathrm{C}$ in $5 \% \mathrm{CO}_{2}$. Unless indicated otherwise, all chemicals were purchased from Sigma-Aldrich (St. Louis, MO, USA).

Ultrasound microbubble-mediated gene transfection. Sprague-Dawley (SD) male rats, 4-weeks of age $(n=25)$, were randomly divided into five groups: group 1, plasmid only; group 2, ultrasound + plasmid; group 3, microbubble + plasmid; group 4, ultrasound + microbubbles + plasmid; and group 5, untreated control. The siMDR1-loaded lipid microbubble was prepared as described (ref?). Plasmid $(50 \mu \mathrm{g})$ in $100 \mu \mathrm{l}$ phosphate-buffered saline (PBS) or lipid microtubule was injected into the tail vein of the experimental rats, and the right testis was exposed to $300 \mathrm{kHz}$-ultrasound irradiation at the acoustic intensity of $2 \mathrm{~W} / \mathrm{cm}^{2}$ for $10 \mathrm{~min}$. After 2 weeks, frozen sections of the right testis were observed. Green fluorescent protein (GFP) expression was considered as an indicator of efficiency of gene delivery.

Real-time PCR analysis. As previously described (14), freshly prepared testis tissues were minced and homogenized in TRIzol reagent and total RNA was extracted by TRIzol method. cDNA templates were generated with $10 \mu \mathrm{g}$ of total RNA and hexamer using SuperScript II reverse transcriptase (Invitrogen). The PCR primers specific for detecting MDR1 (sense, 5'-GAGAACATCGCCTACGG-3' and antisense, 5'-GCTTCCTGGACGACCTT-3'); and GAPDH (sense, 5'-TGGATGGTCCCTCTGGAA-3' and antisense, 5'-GTGAG CTTCCCGTTCAGC-3') were designed using the Primer 3.0 program. Real-time PCR reactions were carried out using a Bio-Rad protocol as follows: $94^{\circ} \mathrm{C}$ for $20 \mathrm{sec}, 55^{\circ} \mathrm{C}$ for $20 \mathrm{sec}$, $70^{\circ} \mathrm{C}$ for $20 \mathrm{sec}$ for 40 cycles, reading plates after each cycle. Data are reported as the fold-change with endogenous GAPDH normalization.

Western blot analysis. Western blotting was performed as previously described $(14,15)$. Freshly prepared testis tissues were homogenized in liquid nitrogen and lysed in RIPA buffer with phenylmethanesulfonyl fluoride (PMSF). Approximately $20 \mu \mathrm{g}$ of total proteins/lane were subjected to $10 \%$ SDS-PAGE gel and then electrically transferred to Immobilon-P membranes (Millipore). The membranes were blocked with $5 \%$ fat-free skimmed milk in Tris-buffered saline and Tween-20 (TBST) buffer at room temperature for
$1 \mathrm{~h}$, followed by incubation with anti-MDR 1 or anti- $\beta$-actin at $4^{\circ} \mathrm{C}$ overnight. After washing with TBST, the membranes were probed with the appropriate secondary antibody conjugated to horseradish peroxidase (Santa Cruz Biotechnology, USA) at room temperature for $1 \mathrm{~h}$. The expression of the proteins was visualized using enhanced chemiluminescent substrate (Kaiji Biotechnology, China) and exposed under the Syngene G:BOX Imaging System.

Daunorubicin accumulation. Daunorubicin accumulation was detected at 2 days after the SD rat treatment. Daunorubicin $(2 \mathrm{ml})$ at a concentration of $0.1 \mathrm{mg} / \mathrm{ml}$ was administered via tail vein injection. Testis tissues were collected after $2 \mathrm{~h}$ and prepared into frozen sections. Red fluorescence which was emitted by daunorubicin indicated the accumulation/concentration of daunorubicin and was detected under a fluorescence microscope.

Establishment of the testicular tumor model. Sixty SD male rats 3-weeks and 3-months of age were collected; 10 rats in each group. After being anesthetized by chloral hydrate, the right testis of the rats was exposed by opening the skin, meat membrane and tunica vaginalis. A cell (10 $\mu \mathrm{l})$ suspension containing $1 \times 10^{6}, 1 \times 10^{7}$ or $1 \times 10^{8}$ cells was injected into the testis via the connection part of the ductuli efferentes testis (16). Then the testis was put back into the scrotum, the spermatic cord and meat membrane were fixed to prevent retraction, and finally the scrotal incision was sutured. The survival and general situation of the rats were dynamically monitored. The testis volume was detected every 7 days to draw a growth curve. Testis was scanned with Color Doppler Ultrasound Diagnostic instrument to observe the change in testicular size, blood supply and sonographic characteristics. Hematoxylin and eosin (H\&E) staining and immunohistochemistry were performed to evaluate tumor progression.

Ultrasound microbubble-mediated siMDR1 transfection combined with chemotherapy in the treatment of testis tumors. Forty SD male rats 3-weeks of age were collected to set up the testicular tumor model as described above. After 3 weeks, successful constructed testicular tumor-bearing rats were randomly divided into 4 groups: group 1, chemotherapy only; group 2, blank microbubbles + ultrasound + chemotherapy; group 3, siMDR1 plasmid-loaded microbubbles + ultrasound + chemotherapy; and group 4, saline control. Microbubbles or siMDR1 plasmid-loaded microbubbles were constantly injected into the tail vein and the right testis was exposed to an ultrasound wave field at $300 \mathrm{kHz}, 2 \mathrm{~W} / \mathrm{cm}^{2}$, irradiation for $10 \mathrm{~min}$. At 1 day after microbubble transfection, the rat models were treated with vincristine chemotherapy by intravenous injection 1 time/week, for 2 weeks. Surface area was calculated on the basis of the Meeh-Rubner formula: $\mathrm{A}\left(\mathrm{m}^{3}\right)=9.1 \times \mathrm{W}(\mathrm{g})^{2 / 3} / 10,000$; rat and human dose conversion formula: trial dose in rats $(\mathrm{mg} / \mathrm{kg})=$ dose in human (mg/kg) x 36/6 (human dose conversion factor/rat dose conversion factor). The survival and general situation of rats was dynamically monitored. The volume of the testis was measured every week. Models were sacrificed at 1 week after chemotherapy for H\&E staining, immunohistochemistry and Fas/p53 gene detection. 
A Group 1
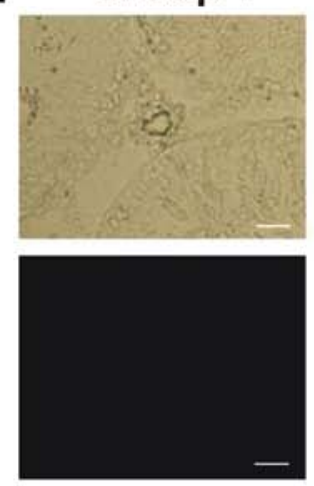

B

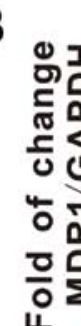

Group 2
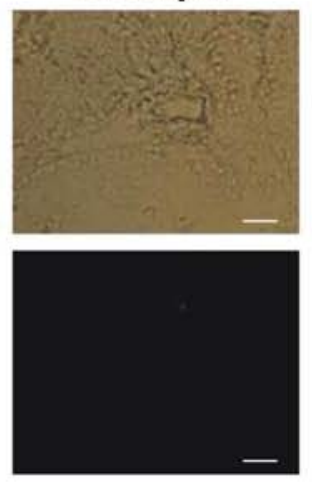

Group 3
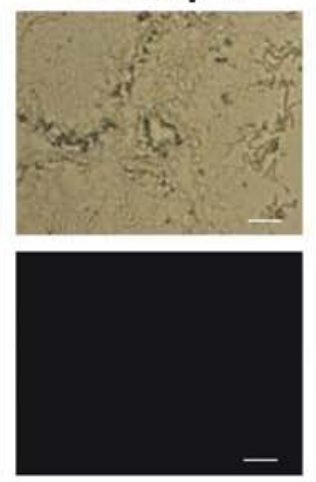

Group 4
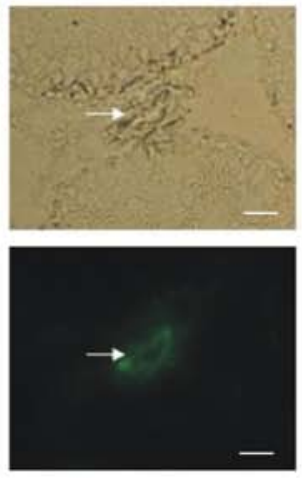

Group 5
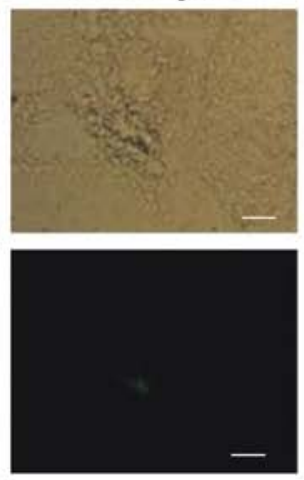

C
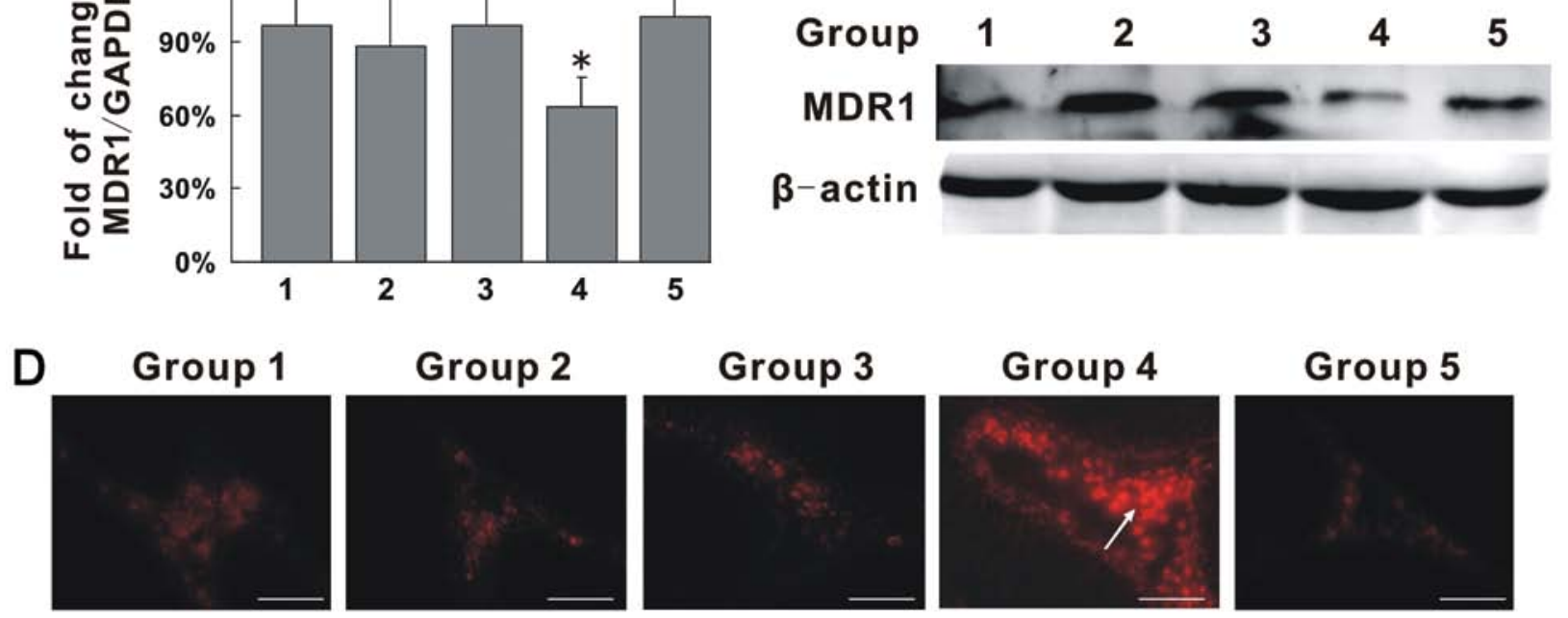

Figure 1. Ultrasound microbubble-mediated gene delivery promotes siMDR1 gene transfection in vivo. Twenty-five Sprague-Dawley male rats 4-weeks of age were randomly divided into five groups: group 1, plasmid only; group 2, ultrasound + plasmid; group 3, microbubble + plasmid; group 4, ultrasound + microbubbles + plasmid; group 5, untreated control. The pSEB-siMDR plasmid, microbubbles or siMDR1-loaded lipid microbubbles were injected into the tail vein of the experimental rats, and the right testis was exposed to ultrasound irradiation. After 2 weeks of treatment, the tissues were harvested for detection. (A) Ultrasound microbubble-mediated gene delivery efficiently promoted the transfection of pSEB-siMDR1 into testicular vascular endothelial cells. GFP expression indicates the efficiency of gene delivery (scale bar, $200 \mu \mathrm{m}$ ). (B) The mRNA expression of the MDR1 gene in testis tissues was detected by realtime PCR. All samples were normalized to GAPDH in triplicate, and the PCR results were confirmed in at least 3 sets of independent experiments ("P<0.05 compared with group 1). (C) The protein expression of MDR1 in testis tissues was detected by western blotting using the anti-MDR1 or $\beta$-actin antibody. Proteins were collected and lysed at 2 weeks after treatment and subjected to SDS-PAGE and western blotting using the anti-MDR 1 antibody. Equal loading of the samples was normalized by $\beta$-actin detection. (D) Daunorubicin accumulation was increased in the tissues treated with siMDR1-loaded lipid microbubble transfection. Red fluorescent cells were observed under a microscope. Cells in group 4 exhibited more red granular fluorescence in the cytoplasm (indicated by a white arrow; scale bar, $200 \mu \mathrm{m})$. GFP, green fluorescent protein.

$H \& E$ staining and immunohistochemistry. The harvested tumor tissues were fixed with $10 \%$ formalin, embedded in paraffin, and serially cut into $5-\mu \mathrm{m}$ sections. Sections of each specimen were stained with H\&E. For immunohistochemistry, the sections were dewaxed in xylene, rehydrated in graded ethanol solutions, and denatured in water at $60^{\circ} \mathrm{C}$ for $1 \mathrm{~h}$, followed by incubation with $\alpha$-1-antitrypsin (ATT) antibody at $4^{\circ} \mathrm{C}$ overnight. After washing in PBS 3 times, the sections were incubated with anti-goat IgG HRP-conjugated secondary antibody at $37^{\circ} \mathrm{C}$ for $1 \mathrm{~h}$, colored with DAB and counterstained with hematoxylin.

Statistical analysis. Quantitative data are presented as the mean \pm SD. Statistical analysis was performed using the two-tailed Student's t-test or analysis of variance. The probability value of $\mathrm{P}<0.05$ was considered to indicate a statistically significant result.

\section{Results}

Ultrasound-targeted microbubble destruction promotes siMDRl gene delivery in vivo. The MDR1 gene which is highly expressed in the testicular capillary wall, hinders chemotherapy drugs into the testis $(4,17)$. Herein, we aimed to use ultrasound-targeted microbubble destruction to deliver the siMDR1 gene into in vivo target testicular capillaries in vivo, to explore whether the expression and function of the MDR1 gene and P-gp were effectively suppressed.

Since the pSEB-siMDR1 plasmid contains the GFP gene sequence, GFP is an indicator to measure the gene transfection efficiency. As shown in Fig. 1A, GFP expression in testicular interstitial capillary endothelial cells, was only observed in the microbubbles + ultrasound group (group 4), suggesting that the pSEB-siMDR1 plasmid was successfully transfected. There was no GFP expression in the other 4 groups. We 

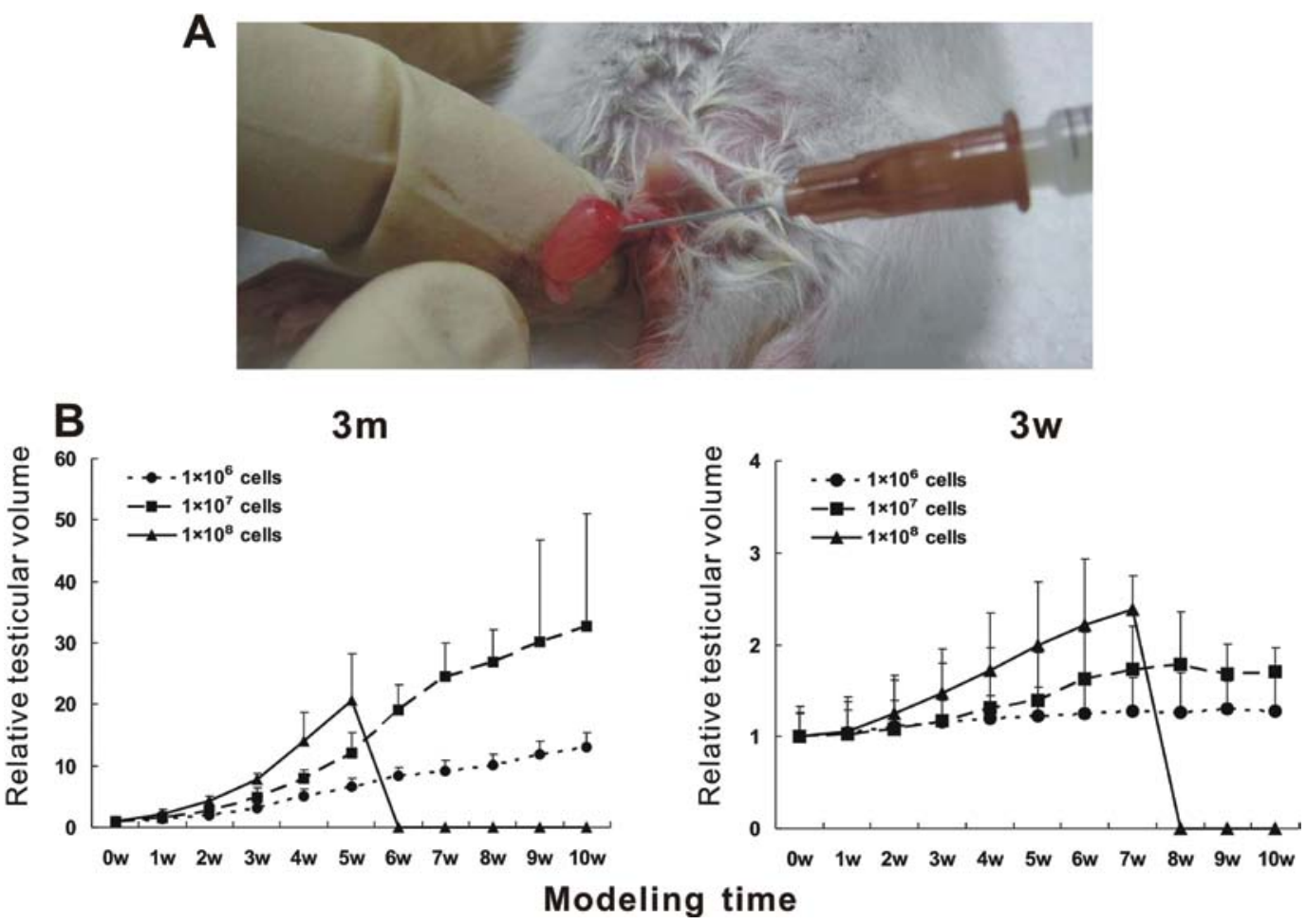

Figure 2. Rats (3-week-old) are suitable for the establishment of the testicular tumor model. (A) Schematic diagram of the operation of the injection of L2RYC cells into the testis tissues. (B) Relative testicular volume of testis tissues injected with different numbers of L2RYC cells. Cells $\left(1 \times 10^{6}\right.$, $1 \times 10^{7}$ or $\left.1 \times 10^{8}\right)$ in $10 \mu \mathrm{l}$ of cell suspension were injected into the testis via the connecting region of the ductuli efferentes testis. Testicular volume was detected every 7 days. $3 \mathrm{~m}$, 3-month-old rats; 3w, 3-week-old rats.

Table I. Testicular tumor formation rate and the survival time of the tumor bearing rats.

Tumor formation/no. Tumor formation/no. Tumor formation/no. Tumor formation/no. Injected No. of after 1-week of surviving rats of surviving rats of surviving rats cell no. samples injection after 3-week after 6-week injection injection injection after 10-week

\begin{tabular}{|c|c|c|c|c|c|c|}
\hline \multirow[t]{3}{*}{ 3-Week-old SD rats } & $1 \times 10^{6}$ & 10 & $0 / 10$ & $0 / 10$ & $0 / 9$ & $0 / 9$ \\
\hline & $1 \times 10^{7}$ & 10 & $6 / 9$ & $9 / 9$ & $7 / 7$ & $2 / 2$ \\
\hline & $1 \times 10^{8}$ & 10 & $8 / 8$ & $4 / 4$ & $0 / 0$ & $0 / 0$ \\
\hline \multirow[t]{3}{*}{ 3-Month-old SD rats } & $1 \times 10^{6}$ & 10 & $0 / 9$ & $0 / 9$ & $0 / 9$ & $0 / 9$ \\
\hline & $1 \times 10^{7}$ & 10 & $1 / 8$ & $1 / 8$ & $1 / 8$ & $0 / 7$ \\
\hline & $1 \times 10^{8}$ & 10 & $9 / 9$ & $7 / 7$ & $2 / 2$ & $0 / 0$ \\
\hline
\end{tabular}

SD, Sprague-Dawley rats.

further demonstrated that the mRNA expression of the MDR1 gene (Fig. 1B) and protein expression (Fig. 1C) of P-gp were reduced in the microbubble + ultrasound group only. No difference was found between the other 3 intervention and control groups. Daunorubicin as a P-gp substrate, spontaneously emits red fluorescence. At $1 \mathrm{~h}$ after daunorubicin injection via tail vein, red fluorescence indicating increased daunorubicin accumulation was observed in the frozen sections of testis in the microbubble + ultrasound group (Fig. 1D), suggesting that the function of P-gp was inhibited and the drug accessed the testis easier. The above results indicated that the combination of ultrasound and microbubbles is an effective method to mediate gene transfection in vivo.
These data suggest that ultrasound microbubble-mediated gene delivery effectively promoted the plasmid DNA transfection in vivo.

Establishment of the testicular tumor model. Rat yolk sac tumor L2 cells were injected into testicular tissue of the SD rats system to establish the testicular tumor model (Fig. 2A), and then we evaluated the model feasibility and application value by observing the biological characteristics of the tumors, growth rate and morphology. In rats of different ages, stages and different planting cell concentrations, the testicular tumor formation rate and the survival time of the tumor-bearing rats were different (Table I). In the group with implanted cells at 

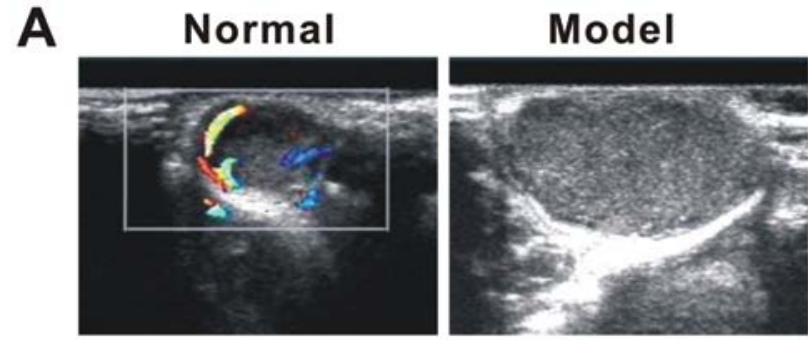

\section{Liquefaction necrosis}

B
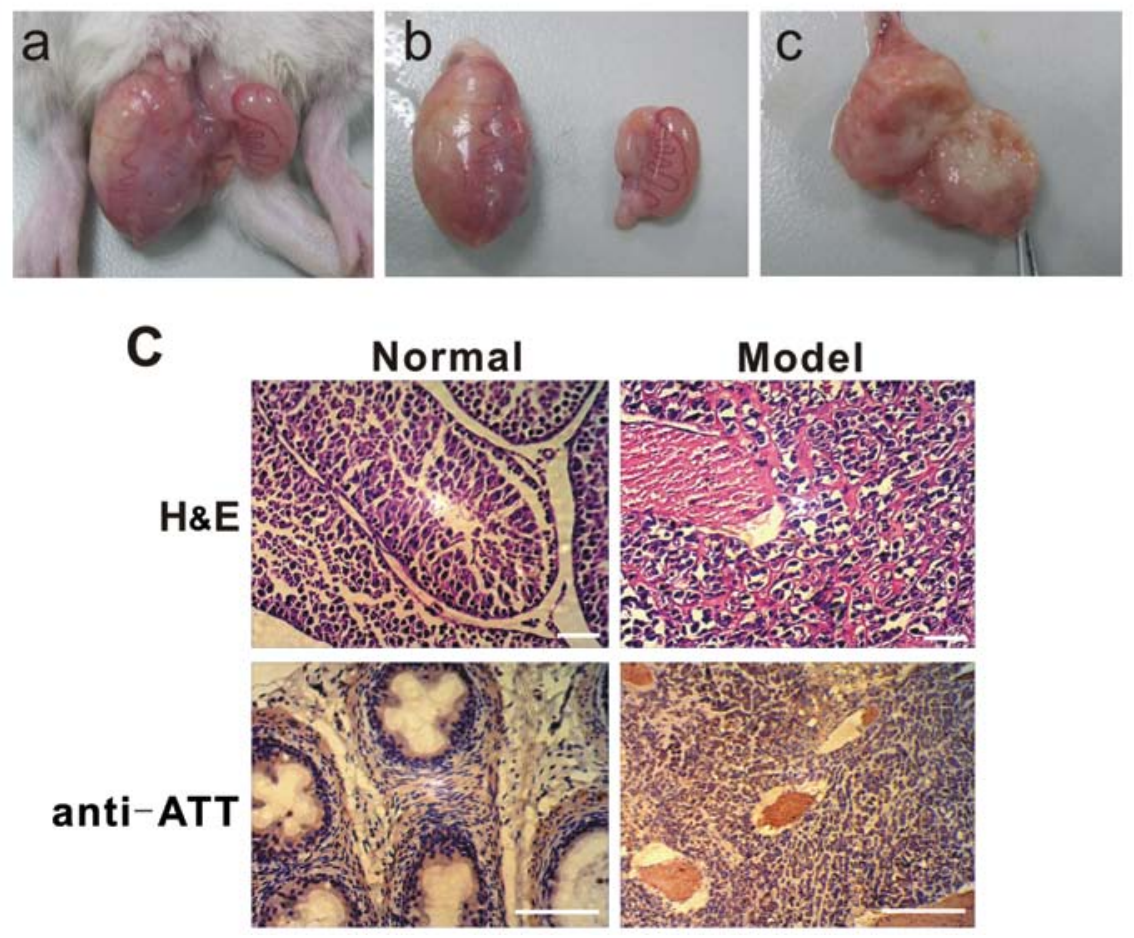

Figure 3. Testicular tumor model was successfully established. L2RYC tumor cells (1x10 $\left.{ }^{7}\right)$ were implanted into testis tissues of 3-week-old SD rats and tumors were detected after 10 weeks. (A) Image of testis tissues using the Color Doppler Ultrasound Diagnostic system. (B) Image of testis tumor tissue. (a) General image of the testis; (b) the volume of testis tumors was much larger than that of the normal testis tissue; (c) cross section of testis tumor tissue. (C) H\&E staining and ATT immunohistochemistry of the normal and tumor testis tissues (scale bar, $200 \mu \mathrm{m}$ ). SD, Sprague-Dawley rats; H\&E, hematoxylin and eosin; ATT, $\alpha$-1-antitrypsin.

the concentration of $1 \times 10^{6} / \mathrm{ml}$, no tumors were present at the end of the experiment. At 3 weeks after implantion of cells at the concentration of $1 \times 10^{7}$, the tumor formation rate of the 3 -week-old rats was $100 \%$, and increased gradually with time after planting, and reached a peak at the 6th week with regions of ulceration and erosion; some rats began to die. The tumor formation rate of the 3 -month-old rats was only $12.5 \%$, and tumors increased more slowly. When plantation of the cells was at the concentration of $1 \times 10^{8}$, the tumor formation rate of both 3-week- and 3-month-old rats were $100 \%$, yet the tumors grew very quickly with a high mortality rate.

The growth curve of testicular tumors (Fig. 2B) showed that the testicular volume of the rats injected with $1 \times 10^{6}$ cells was similar to that of the control group. With injection of $1 \times 10^{7}$ tumor cells, the relative volume of the testis was significantly larger than that of the control group, particularly the 3-week-old rats. With injection of $1 \times 10^{8}$ tumor cells, the testis volume increased rapidly; all rats died after 5 weeks in the 3 -weekold rats and 7 weeks in the 3-month-old SD rats, respectively. Thus, we chose to establish the testicular tumor model with the 3-week-old rats and implanted cells at the concentration of $1 \times 10^{7}$. Ultrasound imaging showed that compared with the control group, the testicular volume of the model side increased significantly; the internal echo was medium and uniform, with small punctuate and cord-like high echo. Part of the testicular tissue exhibited liquefied necrosis with no echo area (Fig. 3A). The affected testis was much larger than the normal side; the testicular mass was suborbicular, cystic and enveloped. Transverse section of the tumor was pale yellow, soft fleshy with partial necrosis (Fig. 3B). Pathological sections under the microscope exhibited loose reticulate structure, regions of adenoid structure, eosinophilic granular and Schiler-Duval bodies by H\&E staining. Immunohistochemistry showed that AAT was positively stained (Fig. 3C). Therefore, the testis tumor model was successfully constructed.

Ultrasound microbubble-mediated siMDR1 gene therapy improves the effect of chemotherapy on the testicular tumors. This experiment aimed to investigate the treatment efficiency of chemotherapy drugs on testicular tumors following the inhibition of P-gp. Following treatment with chemotherapy drugs, tumor growth was inhibited. The survival rate was 

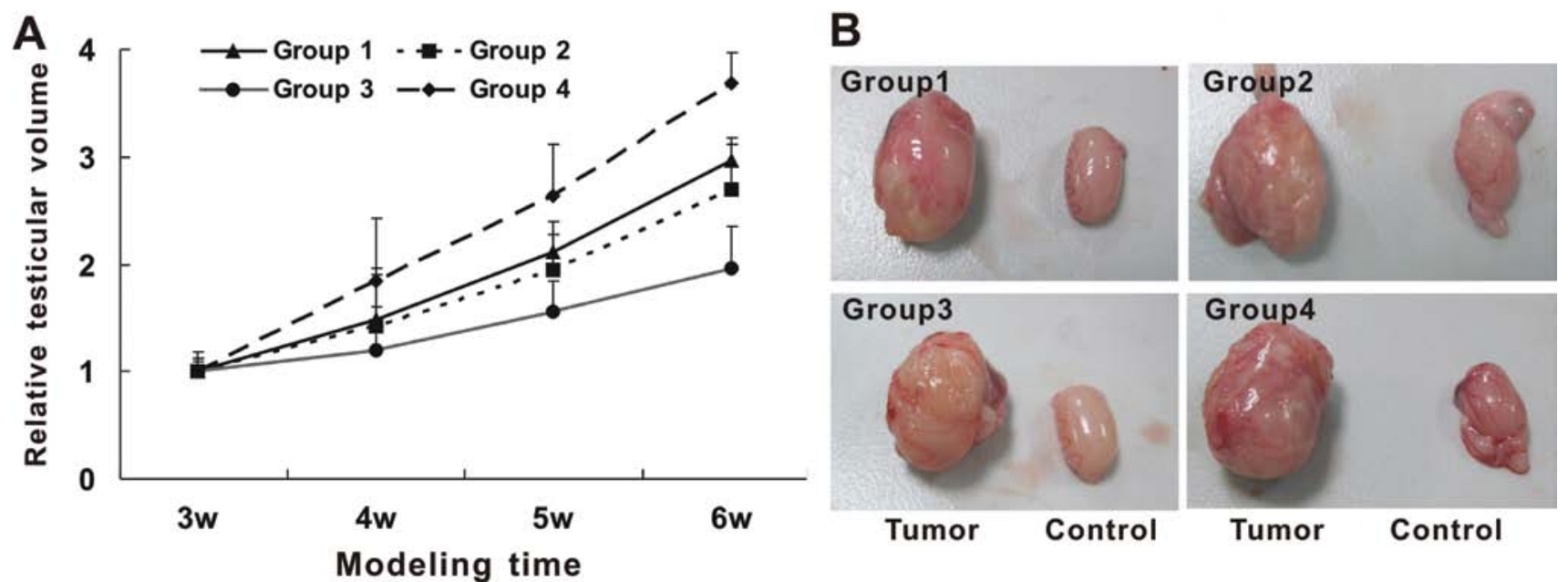

Figure 4. Ultrasound microbubble-mediated siMDR1 gene therapy effectively promotes the effect of chemotherapy on testicular tumors. Testicular tumor model rats were treated with group 1, chemotherapy only; group 2, blank microbubbles + ultrasound + chemotherapy; group 3, siMDR1 plasmid loaded microbubbles + ultrasound + chemotherapy; group 4, saline control. (A) Relative testicular volume of the different treatment groups. (B) Testicular volume of the tumors compared with the controls at 6 weeks.

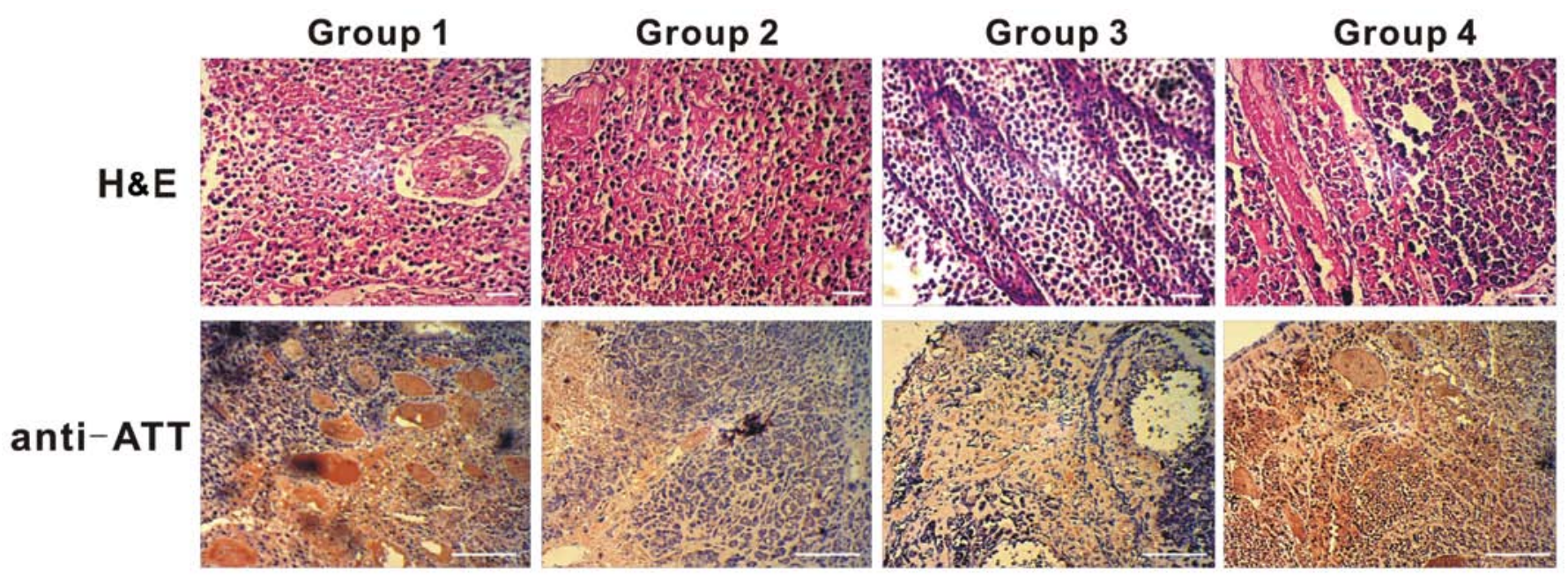

Figure 5. Ultrasound microbubble-mediated siMDR1 gene therapy effectively improves the pathological changes in the testicular tumor tissues. Testicular tumor model rats were treated as described in Fig. 4. H\&E staining and ATT immunohistochemistry of the different treated testis tumor tissues were carried out at 6 weeks of the modeling treatment process (scale bar, $200 \mu \mathrm{m}$ ). H\&E, hematoxylin and eosin; ATT, $\alpha$-1-antitrypsin.

improved obviously when compared with the control group at the same time-point. At the end-point, the survival rate of group 3 was increased to $60 \%$, which was significantly different with the other groups $(\mathrm{P}<0.05)$. Chemotherapy was reported to inhibit the growth of tumors (18). Relative testis volume was significantly smaller than that of the control group. In our groups, the testicular volume of the ultrasound microbubble-mediated siMDR1 therapy after chemotherapy was the smallest (Fig. 4A and B), indicating the best efficiency of tumor suppression.

H\&E staining showed that the numbers of tumor cells within the testicular tissues of group 1-3 were significantly decreased compared with that of group 4, without tumorspecific adenoid structure, and no eosinophilic bodies and Schiler-Duval bodies. Particularly group 3 had visible lumenlike structure in testis tissues. Immunohistochemistry results showed that positive AAT expression of testicular tissues in group 1-3 was lower than that in group 4 (Fig. 5). Therefore, we further confirmed that ultrasound combined with micro- bubble transfection of siMDR1 in the testicular capillary wall provided easier access of vincristine to the testis tissues, and thus improved the effect of chemotherapy on testicular tumors.

\section{Discussion}

Testicular cancer is one of the most common types of cancer of the urinary and reproductive system. The effect of chemotherapy still requires improvement $(1,2,19,20)$. The blood-testis barrier hinders chemotherapy drugs into the testis tissues, which is an important factor that affects treatment outcome $(21,22)$. A recent study has found that an ATP-dependent drug efflux pump protein is expressed in the capillary endothelium of the testis and is related to the formation of the blood-testis barrier (23). Previous studies have demonstrated that this protein associated with tumor multidrug-resistance is P-glycoprotein (P-gp), which is strongly expressed in testicular interstitial capillary endothelial cells (24). P-gp is a glycoprotein encoded by 
the MDR1 gene, is located on the cell membrane and is an ATP-dependent efflux pump, which pumps the insoluble toxic substances out of the cell, so that chemotherapy drugs cannot easily enter into the testis $(4,5,25)$. In an MDR1-knockout rat model, the concentration of $\mathrm{P}$-gp substrates in the testis was significantly higher than that in the normal rat (26). The structure of the blood-brain barrier is similar to the bloodtestis barrier. The rate of positive P-gp expression was found to be $65.8 \%$ in the brain tissues of children with intracranial tumors, while the positive rate of P-gp in brain tissues of children with non-intracranial tumors was only $10 \%$ (27). Hendrikse et al (28) used isotype-labeled P-gp reversal agents to block the P-gp function in the blood-brain barrier, and found that the concentration of intracranial drug increased by 13 -fold, indicating that P-gp plays an important role in the blood-brain barrier, and possible also in the blood-testis barrier. P-gp-mediated reverse efflux is not only a part of the biological function of the blood-testis barrier, yet also may be associated with the application of chemotherapy drugs in testicular tumors. Therefore, breaking through the barrier has a great effect on the treatment of testicular cancer $(5,8,29,30)$.

In the present study, we aimed to use RNA interference technology to silence the mRNA expression of the endogenous MDR1 gene, resulting in inhibition of P-gp expression and resistance reversal in testicular tumors, providing a suitable condition for chemotherapy drug treatment. The main concern of in vivo gene therapy is how to successfully transfect and express the objective gene in target cells (31). In recent studies, it has been demonstrated that ultrasound-mediated gene transfer is a safe, efficient and non-invasive gene therapy as a possible alternative to viral gene transfer. It plays a significant role in gene therapy-based approaches to the treatment of diseases (32-34). We previously demonstrated that ultrasound microbubble-mediated destruction could effectively delivery siRNA specific for the MDR1 gene into L2-RYC cells using ultrasound microbubble-mediated destruction. We successfully inhibited MDR1 expression and function of P-gp. L2-RYC cells with MDR1 silencing became more sensitive to anticancer drugs, vincristine and dactinomycin (9). In the present study, we further confirmed that ultrasound microbubble-mediated destruction led to transfection of pSEB-siMDR1 into rat testis capillary endothelial cells. The endogenous expression of the MDR1 gene and P-protein decreased. P-gp function was also suppressed, altering the high-resistance state of testicular tumors in response to chemotherapy drugs.

Since the origin and classification of testicular tumors are markedly complex, the animal models are not, strictly speaking, valid for the study of testicular tumors. Usually the transplanted tumor model is established in nude rats; however, nude rats have characteristics of immunodeficiency, therefore the biological characteristics of testicular tumors are different compared with normal immune rats (35). In this study, we implanted a rat yolk sac tumor cell line.

L2RYC cells were injected into the testis tissues of normal immune system SD rats to establish the animal model. Graft rejective reaction is the most important problem of a cell implanted animal model and the use of immunosuppressants is needed. However, in tumor therapy, immunosuppressants are also used as antitumor medicines. The efficiency of chemotherapeutics on tumors may be affected using immuno- suppressants (36-38). Thus, in the present experiment, we used 3-week- and 3-month-old SD rats to set-up the animal model. The establishment of a cell transplantation tumor model prior to development of an adaptive immune system may be feasible.

The number of inoculated cells is another important factor affecting the establishment of a transplanted tumor model (39). Usually in the nude rat model, $1 \times 10^{6}$ inoculated cells form a tumor (40). However, no tumor formation was found in the 3-week- or 3-month-old SD rats following $1 \times 10^{6} \mathrm{~L} 2 \mathrm{RYC}$ cell transplantation. When $1 \times 10^{7}$ cells were implanted into the testis, tumors were formed in the 3-week-old rats but not in the 3-month-old rats. This result may be associated with host immune graft rejection suggesting that more cells are required to establish the xenografts in normal animals compared to the nude rats. If the number of cells was excessive $\left(1 \times 10^{7}\right)$, tumor cells were strongly invasive, leading to the quick death of the host rat. The pathological manifestations mainly exhibited testicular necrosis, which was different from the clinical course and performance, and not suitable for tumor study. At 4 weeks after $1 \times 10^{7} \mathrm{~L} 2 \mathrm{RYC}$ cells were inoculated in the 3-week-old SD rats, ultrasound imaging and histopathological characteristics were similar to clinical testicular yolk sac tumors, indicating that this method can successfully establish a feasible model of testicular tumors.

Next, we assessed the feasibility of ultrasound microbubble-mediated destruction in delivering chemotherapy drugs in the tumor model. By intravenous injection of microbubbles, the permeability of the cell membrane was enhanced under local ultrasonic action, thus promoting the transfection of target genes or drugs (41). Through the observation of tumor growth, the survival rate of tumor-bearing rats, and detection of pathological changes, we found that ultrasound microbubblemediated destruction followed by chemotherapy treatment was most effective for the treatment of testis tumors. Compared with the chemotherapy alone group, ultrasound microbubblemediated destruction combined with chemotherapy had a better effect, probably since the permeability of testicular capillary transiently increased under ultrasound microbubble-mediated destruction. The drug concentration in the testis was slightly higher than that in the chemotherapy alone group. Expression of an exogenous gene mediated by transfection sustains for 2-3 weeks (42). MDR1 expression was suppressed during this time window. The permeability of the testicular vascular was selectively increased following suppression of the P-gp substrate, and then drugs easily entered into the testis tissues, thereby enhancing the effect of chemotherapy.

In summary, the present study mainly focused on the issue that chemotherapy drugs cannot easily enter testicular tumors. We used ultrasound microbubble-mediated destruction method to increase the permeability of the capillary endothelial cell membrane, and achieved efficient in vivo transfection of the siMDR1 gene and inhibited P-gp production. The reversal of chemotherapy drug resistance in testicular tumors improves the treatment effect, which is expected to provide an effective method for the treatment of testicular tumors.

\section{Acknowledgements}

The present study was supported in part by a research grant from the National Natural Science Foundation of China (nos. 
81001030 to Y.H. and 81301300 to X.-J.J.). This study was also supported in part by the Special Fund of Chongqing Key Laboratory (CSTC) (to G.-H.W.), Program for Innovation Team Building at Higher Education Institutions in Chongqing, China (to G.-H.W.) and National Clinical Key Subject Construction Project (AXA medical office letter [2013]544).

\section{References}

1. Nakamura T and Miki T: Recent strategy for the management of advanced testicular cancer. Int J Urol 17: 148-157, 2010.

2. Allaway M, Nseyo UO and Kandzari SJ: Primary testicular sarcoma. J Urol 163: 1871, 2000.

3. Kawai $\mathrm{K}$ and Akaza H: Current status of chemotherapy in riskadapted management for metastatic testicular germ cell cancer. Cancer Sci 101: 22-28, 2010.

4. Schrader AJ, Seger M, Konrad L, Olbert P, Hegele A, Hofmann R and Heidenreich A: Clinical impact of MDR1-expression in testicular germ cell cancer. Exp Oncol 29: 212-216, 2007.

5. Bart J, Hollema H, Groen HJ, de Vries EG, Hendrikse NH, Sleijfer DT, Wegman TD, Vaalburg W and van der Graaf WT: The distribution of drug-efflux pumps, P-gp, BCRP, MRP1 and MRP2, in the normal blood-testis barrier and in primary testicular tumours. Eur J Cancer 40: 2064-2070, 2004.

6. Kimura Y, Matsuo M, Takahashi K, Saeki T, Kioka N, Amachi T and Ueda K: ATP hydrolysis-dependent multidrug efflux transporter: MDR 1/P-glycoprotein. Curr Drug Metab 5: 1-10, 2004.

7. Sivapackiam J, Harpstrite SE, Prior JL, Gu H, Rath NP and Sharma V: Synthesis, molecular structure, and validation of metalloprobes for assessment of MDR1 P-glycoprotein-mediated functional transport. Dalton Trans 39: 5842-5850, 2010.

8. Bart J, Groen HJ, van der Graaf WT, Hollema H, Hendrikse NH, Vaalburg W, Sleijfer DT and de Vries EG: An oncological view on the blood-testis barrier. Lancet Oncol 3: 357-363, 2002.

9. He Y, Bi Y, Hua Y, Liu D, Wen S, Wang Q, Li M, Zhu J, Lin T, He D, et al: Ultrasound microbubble-mediated delivery of the siRNAs targeting MDR1 reduces drug resistance of yolk sac carcinoma L2 cells. J Exp Clin Cancer Res 30: 104, 2011.

10. Shi Z, Liang YJ, Chen ZS, Wang XW, Wang XH, Ding Y, Chen LM, Yang XP and Fu LW: Reversal of MDR1/P-glycoprotein-mediated multidrug resistance by vector-based RNA interference in vitro and in vivo. Cancer Biol Ther 5: 39-47, 2006.

11. Yin H, Kanasty RL, Eltoukhy AA, Vegas AJ, Dorkin JR and Anderson DG: Non-viral vectors for gene-based therapy. Nat Rev Genet 15: 541-555, 2014.

12. Carson AR, McTiernan CF, Lavery L, Hodnick A, Grata M, Leng X, Wang J, Chen X, Modzelewski RA and Villanueva FS: Gene therapy of carcinoma using ultrasound-targeted microbubble destruction. Ultrasound Med Biol 37: 393-402, 2011.

13. Carson AR, McTiernan CF, Lavery L, Grata M, Leng X, Wang J, Chen $\mathrm{X}$ and Villanueva FS: Ultrasound-targeted microbubble destruction to deliver siRNA cancer therapy. Cancer Res 72 6191-6199, 2012

14. Bi Y, He Y, Huang J, Su Y, Zhu GH, Wang Y, Qiao M, Zhang BQ, Zhang H, Wang Z, et al: Functional characteristics of reversibly immortalized hepatic progenitor cells derived from mouse embryonic liver. Cell Physiol Biochem 34: 1318-1338, 2014

15. Bi Y, Gong M, He Y, Zhang X, Zhou X, Zhang Y, Nan G, Wei X, Liu Y, Chen J, et al: AP2 $\alpha$ transcriptional activity is essential for retinoid-induced neuronal differentiation of mesenchymal stem cells. Int J Biochem Cell Biol 46: 148-160, 2014.

16. Nettersheim D, Westernströer B, Haas N, Leinhaas A, Brüstle O, Schlatt S and Schorle H: Establishment of a versatile seminoma model indicates cellular plasticity of germ cell tumor cells. Genes Chromosomes Cancer 51: 717-726, 2012.

17. Sakata S, Fujiwara M, Ohtsuka K, Kamma H, Nagane M, Sakamoto A and Fujioka Y: ATP-binding cassette transporters in primary central nervous system lymphoma: Decreased expression of MDR 1 P-glycoprotein and breast cancer resistance protein in tumor capillary endothelial cells. Oncol Rep 25: 333-339, 2011.

18. Jin H, Yang R, Ross J, Fong S, Carano R, Totpal K, Lawrence D, Zheng Z, Koeppen H, Stern H, et al: Cooperation of the agonistic DR5 antibody apomab with chemotherapy to inhibit orthotopic lung tumor growth and improve survival. Clin Cancer Res 14: 7733-7740, 2008.

19. Hanna NH and Einhorn LH: Testicular cancer - discoveries and updates. N Engl J Med 371: 2005-2016, 2014.
20. O'Reilly A, MacEneaney P, Mayer N, O'Reilly SP and Power DG: Testicular cancer and platinum: A double-edged sword. J Clin Oncol 32: e46-e48, 2014

21. Chang XB: A molecular understanding of ATP-dependent solute transport by multidrug resistance-associated protein MRP1. Cancer Metastasis Rev 26: 15-37, 2007.

22. Su L, Mruk DD and Cheng CY: Drug transporters, the blood-testis barrier, and spermatogenesis. J Endocrinol 208: 207-223, 2011.

23. Klein DM, Wright SH and Cherrington NJ: Localization of multidrug resistance-associated proteins along the blood-testis barrier in rat, macaque, and human testis. Drug Metab Dispos 42: 89-93, 2014.

24. Melaine N, Liénard MO, Dorval I, Le Goascogne C, Lejeune H and Jégou B: Multidrug resistance genes and P-glycoprotein in the testis of the rat, mouse, Guinea pig, and human. Biol Reprod 67: 1699-1707, 2002.

25. Guo $\mathrm{C}$ and Jin X: Chemoprotection effect of multidrug resistance 1 (MDR1) gene transfer to hematopoietic progenitor cells and engrafted in mice with cancer allows intensified chemotherapy. Cancer Invest 24: 659-668, 2006.

26. Uhr M, Steckler T, Yassouridis A and Holsboer F: Penetration of amitriptyline, but not of fluoxetine, into brain is enhanced in mice with blood-brain barrier deficiency due to mdrla P-glycoprotein gene disruption. Neuropsychopharmacology 22: 380-387, 2000.

27. Guo Z, Zhu J, Zhao L, Luo Q and Jin X: Expression and clinical significance of multidrug resistance proteins in brain tumors. J Exp Clin Cancer Res 29: 122, 2010.

28. Hendrikse NH, de Vries EG, Eriks-Fluks L, van der Graaf WT, Hospers GA, Willemsen AT, Vaalburg W and Franssen EJ: A new in vivo method to study P-glycoprotein transport in tumors and the blood-brain barrier. Cancer Res 59: 2411-2416, 1999.

29. Dave DS, Leppert JT and Rajfer J: Is the testis a chemo-privileged site? Is there a blood-testis barrier? Rev Urol 9: 28-32, 2007.

30. França LR, Auharek SA, Hess RA, Dufour JM and Hinton BT: Blood-tissue barriers: Morphofunctional and immunological aspects of the blood-testis and blood-epididymal barriers. Adv Exp Med Biol 763: 237-259, 2012.

31. Guo X and Huang L: Recent advances in nonviral vectors for gene delivery. Acc Chem Res 45: 971-979, 2012.

32. Panje CM, Wang DS and Willmann JK: Ultrasound and microbubble-mediated gene delivery in cancer: Progress and perspectives. Invest Radiol 48: 755-769, 2013.

33. Sirsi SR and Borden MA: Advances in ultrasound mediated gene therapy using microbubble contrast agents. Theranostics 2: 1208-1222, 2012.

34. Castle J, Butts M, Healey A, Kent K, Marino M and Feinstein SB: Ultrasound-mediated targeted drug delivery: Recent success and remaining challenges. Am J Physiol Heart Circ Physiol 304: H350-H357, 2013.

35. Ortiz RJ, Lizama C, Codelia VA and Moreno RD: A molecular evaluation of germ cell death induced by etoposide in pubertal rat testes. Mol Hum Reprod 15: 363-371, 2009.

36. Jing H, Lin SZ and Yang X: Synergistic effect of emodin and cyclosporine $A$ on rejective reaction against liver graft in rats. Zhongguo Zhong Xi Yi Jie He Za Zhi 28: 614-616, 2008 (In Chinese).

37. Lin SZ, Chen KJ, Tong HF, Jing H, Li H and Zheng SS: Emodin attenuates acute rejection of liver allografts by inhibiting hepatocellular apoptosis and modulating the Th1/Th2 balance in rats. Clin Exp Pharmacol Physiol 37: 790-794, 2010.

38. Lawrance IC: Topical agents for idiopathic distal colitis and proctitis. J Gastroenterol Hepatol 26: 36-43, 2011.

39. van den Engel NK, Rüttinger D, Rusan M, Kammerer R, Zimmermann W, Hatz RA and Winter H: Combination immunotherapy and active-specific tumor cell vaccination augments anti-cancer immunity in a mouse model of gastric cancer. J Transl Med 9: 140, 2011.

40. He LF, Wang TT, Gao QY, Zhao GF, Huang YH, Yu LK and Hou YY: Stanniocalcin-1 promotes tumor angiogenesis through up-regulation of VEGF in gastric cancer cells. J Biomed Sci 18: 39, 2011.

41. Park K: Ultrasound-activatable drug-loaded microbubbles for intracellular targeting. J Control Release 132: 151, 2008.

42. Deng F, Chen X, Liao Z, Yan Z, Wang Z, Deng Y, Zhang Q, Zhang Z, Ye J, Qiao M, et al: A simplified and versatile system for the simultaneous expression of multiple siRNAs in mammalian cells using Gibson DNA Assembly. PLoS One 9: e113064, 2014. 